'Departamento de Inmunología Clínica y Reumatología, Pontificia Universidad Católica de Chile. Santiago, Chile. ${ }^{2}$ Servicio de Dermatología, Hospital Sótero del Río. Santiago, Chile.

3Unidad de Inmunología, Clínica Las Condes. Santiago, Chile.

${ }^{4}$ Unidad de Reumatología e Inmunología, Hospital Militar de Santiago. Santiago, Chile. ${ }^{5}$ Unidad de Inmunología y Alergias, Clínica Santa María. Santiago, Chile.

${ }^{6}$ Departamento de Dermatología Facultad de Medicina Universidad de Chile. Santiago, Chile. Departamento de Dermatología, Clínica Las Condes. Santiago, Chile.

Todos los autores colaboraron en igual forma en la elaboración del documento.

Los autores declaran no tener conflicto de interés. Financiamiento: FONDECYT 11161102.

Recibido el 13 de febrero de 2018, aceptado el 30 de agosto de 2018.

Correspondencia a: Raquel Aguilera-Insunza Diagonal Paraguay 362, Santiago, Santiago, Chile reaguilera@uc.cl

Material suplementario que puso al final del texto.

\section{Guía clínica chilena de urticaria crónica espontánea}

\author{
RAQUEL AGUILERA-INSUNZA ${ }^{1}$, HERNÁN CORREA², \\ CAROLINA DÍAZ ${ }^{3,4}$, MARÍA ANGÉLICA MARINOVIC ${ }^{5}$, \\ FERNANDO VALENZUELA ${ }^{6,7}$
}

\section{Chilean guidelines for chronic urticaria}

Chronic urticaria (CU) is characterized by itchy wheals, angioedema or both lasting six weeks or more. It is classified as spontaneous (CSU) and inducible urticaria (CIndU), depending whether there is an identifiable trigger or not. CSU is the predominant form, affecting more than $75 \%$ of patients, although overlaps often occur. The prevalence of CSU throughout life is around $1.8 \%$ and predominates in women at a ratio of $2: 1$. The higher incidence of CSU is between 20 and 40 years of age and lasts between one to five years. However, in up to $20 \%$ of patients the disease may last longer. CSU not only hampers quality of life, but also affect performance at work and school. The diagnosis of CSU is mainly clinical, and laboratory tests are required depending on the clinical history of the patient. Extensive laboratory tests are usually unnecessary. Second generation anti-histamines are used as first line treatment for CSU treatment. In refractory patients, systemic treatments, such as cyclosporine or omalizumab are suggested. We herein report the first Chilean CSU guidelines.

(Rev Med Chile 2018; 146: 1334-1342)

Key words: Angioedema; Guideline; Pruritus; Urticaria.
L a urticaria se define como la aparición repentina de habones pruriginosos, angioedema o ambos. El habón corresponde a una elevación circunscrita de la piel, de forma y dimensiones variables, con un área edematosa central rodeado por una zona eritematosa (Figura 1$)^{1}$.

El angioedema corresponde a un aumento de volumen localizado, que compromete la dermis profunda, tejido subcutáneo y submucoso, secundario al aumento de la permeabilidad vascular (Figura 2) ${ }^{2}$. Cuando se asocia a urticaria, afecta principalmente la región palpebral, labios y/o cara, sin compromiso de la vía aérea en la mayoría de los $\operatorname{casos}^{2}$. El angioedema es más bien doloroso y su resolución es más lenta que el habón, pudiendo durar hasta $72 \mathrm{~h}^{1}$. Por lo general se presenta en forma concomitante a los habones, sin embargo,
10 a $20 \%$ de los pacientes puede presentarlo en forma aislada ${ }^{2}$.

Según su duración, la urticaria se clasifica en aguda (menos de 6 semanas) y crónica (más de 6 semanas) (Tabla 1$)^{1}$. La urticaria crónica (UC) se clasifica a su vez en UC espontánea (UCE) y UC inducible (UCInd), dependiendo si existe o no un estímulo definido (Tabla 1). La UCE es la forma predominante, afectando a más de $75 \%$ de los pacientes, aunque ambos tipos pueden coexistir en un mismo paciente ${ }^{3}$.

La prevalencia de UC a lo largo de la vida es alrededor de $1,8 \%$ y es más frecuente en mujeres, a razón de $2: 1^{4}$. Si bien puede afectar a cualquier edad, la incidencia máxima se observa entre los 20 y 40 años $^{4}$. La UCE es una enfermedad crónica con una duración entre 1 a 5 años en la mayoría 


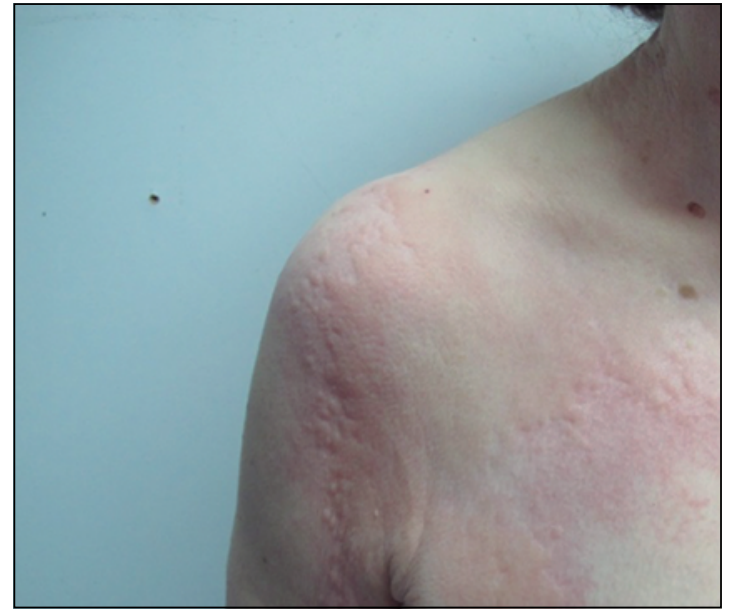

Figura 1. Foto de habón urticarial. Foto con consentimiento de parte del paciente para su uso para esta publicación.

de los casos, estimándose que 50\% mejorarán los primeros 6 meses, $20 \%$ en los primeros 3 años, $20 \%$ entre los 5 y 10 años, y menos de $2 \%$ en 25 años ${ }^{4}$. Se ha visto que la UCE es más prolongada en casos de enfermedad más severa, y cuando se asocia con angioedema y/o a UCInd ${ }^{4}$.

\section{Diagnóstico}

El diagnóstico de UCE es clínico y el laboratorio debe estar orientado a cada paciente, sin embargo, para descartar patologías sistémicas, se recomiendan algunos exámenes básicos en todos los pacientes (Tabla 2$)^{5}$.

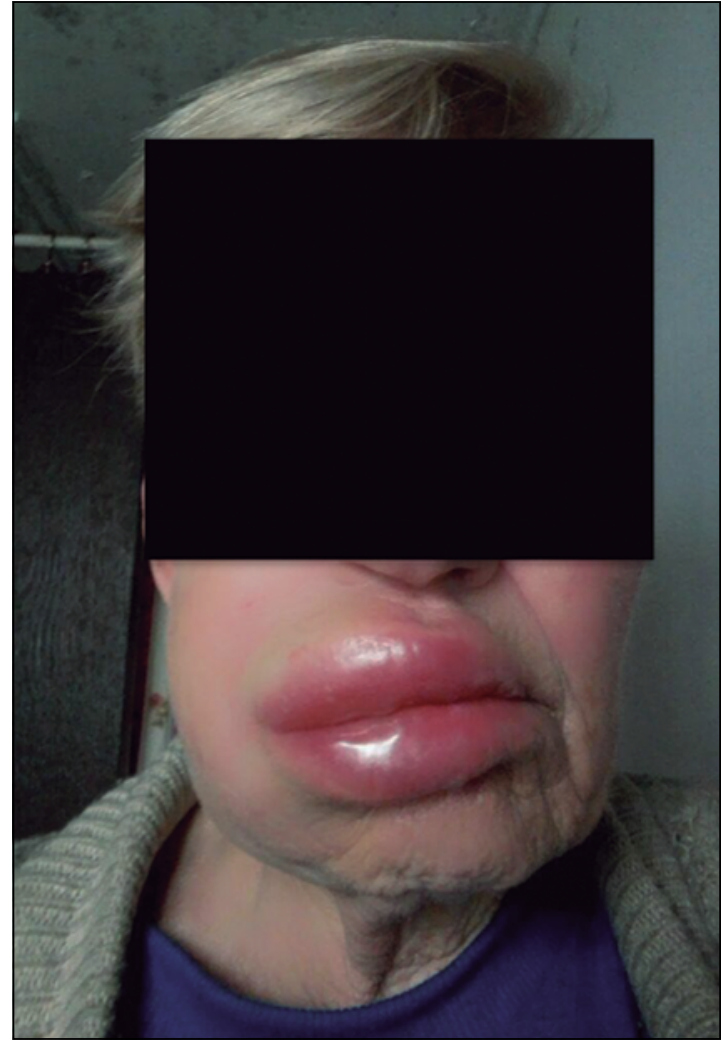

Figura 2. Foto de angioedema de labios. Foto con consentimiento de parte del paciente para su uso para esta publicación.

En casos de UCE de larga data o síntomas más severos, se sugieren además exámenes extendidos para descartar otras patologías asociadas (Tabla $2)^{5}$.

\section{Tabla 1. Clasificación de subtipos de urticaria crónica}

\begin{tabular}{|lll|}
\hline Subtipos de urticaria crónica & & \\
Urticaria crónica espontánea & $\begin{array}{l}\text { Aparición espontánea de habones, angioedema o ambos por } \geq 6 \text { semanas, sin causa } \\
\text { conocida }\end{array}$ \\
\hline Urticaria inducible & Subgrupo & Desencadenante \\
& Dermografismo sintomático & Frotación mecánica \\
& Urticaria por frío & Contacto con aire, agua o sólidos fríos \\
& Urticaria por calor & Contacto con aire, agua o sólidos calientes \\
& Urticaria por presión retardada & Presión vertical sostenida \\
& Angioedema vibratorio & Vibración \\
& Urticaria solar & Radiación UV y/o luz visible \\
& Urticaria de contacto & Contacto con un estímulo alergénico o no alergénico \\
& Urticaria colinérgica & Incremento de la temperatura corporal central \\
& Urticaria acuagénica & Contacto con agua a cualquier temperatura \\
\hline
\end{tabular}


Tabla 2. Exámenes diagnósticos en UCE

\begin{tabular}{|c|c|c|}
\hline Tipo de evaluación & Examen & Observaciones \\
\hline Exámenes de rutina & $\begin{array}{l}\text { - Hemograma completo } \\
\text { - VHS } \\
\text { - Anticuerpos anti-nucleares (ANA) }\end{array}$ & \\
\hline $\begin{array}{l}\text { Evaluación con estudio } \\
\text { extendido }\end{array}$ & $\begin{array}{l}\text { - Hormona estimulante de tiroides (TSH) } \\
\text { - Anticuerpos antitiroideos } \\
\text { - Proteína C reactiva (PCR) } \\
\text { - Estudio de enfermedades infecciosas (p. Ej: VIH, sífilis) } \\
\text { - Coproparasitológico seriado } \\
\text { - Coprocultivo }\end{array}$ & Según la patología del paciente \\
\hline Evaluación de la severidad & $\begin{array}{l}\text { - } \text { Ige total } \\
\text { - Dímero-D } \\
\text { - } \text { Test de suero autólogo }\end{array}$ & \\
\hline $\begin{array}{l}\text { Evaluación diagnóstico } \\
\text { diferencial }\end{array}$ & $\begin{array}{l}\text { - Triptasa sérica basal } \\
\text { - Electroforesis de proteínas séricas } \\
\text { - Biopsia de piel }\end{array}$ & $\begin{array}{l}\text { Sospecha de mastocitosis } \\
\text { Sobre los } 40 \text { años de edad } \\
\text { Sospecha de urticaria vasculítica }\end{array}$ \\
\hline
\end{tabular}

Otros marcadores de laboratorio que han demostrado utilidad en la evaluacuión de UCE comprenden el Dímero-D y el test de suero autólogo (TSA). Niveles elevados de dímero-D, como consecuencia de la activación de la vía extrínseca de la cascada de la coagulación, se asocian a UCE de mayor duración y severidad ${ }^{6}$. Además se ha visto que éste disminuye a medida que mejora la enfermedad ${ }^{6}$.

El TSA consiste en una intradermorreacción con suero autólogo del paciente. Un resultado positivo indica acivación de mastocitos mediada por auto-anticuerpos o factores séricos. La positividad del TSA se ha asociado a enfermedad más prolongada y con peor respuesta a tratamiento ${ }^{7}$.

Debido a que esta no es una enfermedad mediada por IgE, no se recomienda realizar test cutáneos a aeroalergenos ni alimentos ${ }^{5}$.

\section{Evaluación de la actividad de la urticaria crónica}

Existen distintas herramientas, basadas en la percepción del paciente, para evaluar la actividad de la enfermedad.

\section{Escala de actividad de la urticaria (UAS)}

Esta escala se basa en una puntuación individual respecto al número de habones e intensidad del prurito durante las últimas $24 \mathrm{~h}$ (tabla suplementaria 1$)^{8}$. Se emplea para medir la actividad de la enfermedad y así evaluar la respuesta a trata- miento ${ }^{8}$. El UAS-7 se calcula sumando el puntaje diario de UAS durante una semana, fluctuando el puntaje entre 0 y 42 (tabla suplementaria 2). Un puntaje $\leq 6$ de UAS-7 refleja una UCE controlada ${ }^{8}$.

\section{Indice de actividad de angioedema (AAS)}

Este índice permite evaluar la actividad de la enfermedad en pacientes con angioedema recurrente y se usa para objetivar la severidad de éste ${ }^{9}$.

La evaluación la realiza el paciente en base a las últimas $24 \mathrm{~h}$ y considera duración, incomodidad física, impacto en la actividad diaria, afectación en la presentación personal y severidad general del angioedema (figura suplementaria 1). Los índices diarios se suman por 7 días para dar un índice semanal o por 4 semanas, que es la forma en que se recomienda para evaluar la evolución del paciente (figura suplementaria 1$)^{9}$.

\section{Test de control de urticaria (UCT)}

Es una herramienta validada y confiable para valorar el nivel de control de la enfermedad, siendo de gran ayuda en las decisiones terapéuticas ${ }^{10}$.

Se basa en preguntas que evalúan el control de signos y síntomas de la enfermedad, así como mejoría de la calidad de vida (QoL), eficacia del tratamiento y control general de la sintomatología (figura suplementaria 2).

El puntaje va entre 0 (sin ninguna mejoría) y 16 (control total del cuadro) (figura suplementaria 2$)^{10}$. 


\section{Diagnóstico diferencial}

El diagnóstico de UCE es eminentemente clínico, sin embargo, hay que considerar eventuales diagnósticos diferenciales, como:

- Urticaria vasculítica: Los habones duran más de $24 \mathrm{~h}$, son urentes, poco pruriginosos y generalmente dejan una pigmentación posterior. Puede acompañarse de fiebre y artralgias ${ }^{11}$.

- Mastocitosis: La forma cutánea se caracteriza por lesiones máculo-papulares de diámetro pequeño, color marrón y aparecen post trauma mínimo de la piel (signo de Darier) ${ }^{12}$.

- Erupción polimorfa del embarazo: Placas eritematosas solevantadas, pruriginosas, que se presentan en el tercer trimestre del embarazo, usualmente en relación a las estrías ${ }^{13}$.

- Eritema multiforme: Cuadro clínico con lesiones cutáneas caracterizadas por anillos eritematosos concéntricos alrededor de un centro pálido (en diana). Las lesiones pueden presentar prurito leve y usualmente duran $7 \mathrm{a}$ 10 días $^{14}$.

- Síndromes autoinflamatorios: Erupción cutánea urticarial que puede acompañarse de síntomas sistémicos, adenopatías, artralgias/artritis y fiebre. Dentro de este grupo se encuentra el Síndrome de Schnitzler, Síndrome de Muckle-Wells, Síndrome autoinflamatorio familiar inducido por frío y Síndrome Crónico Infantil Neurológico Cutáneo y Articular ${ }^{14,15}$.

- Angioedema recurrente hereditario o adquirido: Angioedema no pruriginoso recurrente sin urticaria asociada muchas veces con compromiso respiratorio y/o intestinal ${ }^{2}$.

\section{Calidad de vida}

El impacto que genera la UCE en el paciente es complejo, afectando no solo la piel sino que también la QoL. La QoL de estos pacientes puede verse afectada por diversas variables, como la imprevisibilidad de los ataques, las restricciones sociales, de vestuario o efectos adversos a medicamentos; determinando un deterioro en la vida privada, social y laboral. Además, el prurito crónico así como el trastorno de sueño secundario serían causantes de comorbilidad psiquiátrica como depresión ${ }^{16}$.

El cuestionario para evaluar QoL en UCE más utilizado es el Cuestionario de calidad de vida dermatológica (DLQI) que permite tener una visión global del impacto en QoL y compara los resultados de estos pacientes con otras patologías cutáneas ${ }^{17}$. Otra herramienta útil y validada al español es el cuestionario de QoL en UC (CUQ2oL), el cual posee una alta especificidad para evaluar la carga que tiene la enfermedad en el paciente (figura suplementaria 3$)^{18,19}$. Por su parte, el cuestionario de QoL en angioedema (AE-QoL) evalúa la respuesta al tratamiento del angioedema desde la mirada de los pacientes ${ }^{20}$.

Existen diversos estudios que han evidenciado el gran deterioro en la QoL de los pacientes que padecen UCE, llegando incluso a niveles mayores que lo producido por otras enfermedades cutáneas, como psoriasis, lepra y dermatitis atópica ${ }^{21}$. Por otro lado, estos pacientes presentan frecuentemente comorbilidades psiquiátricas como ansiedad, depresión y trastornos somatomorfos ${ }^{22}$. Sumado a esto, se ha visto que la QoL está aún más reducida en los pacientes que tienen UCE y además diagnóstico psiquiátrico ${ }^{16}$.

\section{Tratamiento de la urticaria crónica espontánea}

El objetivo de la terapia en UCE es lograr el control completo de los síntomas con un tratamiento lo más seguro posible. Este se basa en dos pilares, la evitación de factores exacerbantes, como el uso de anti-inflamatorios no esteroidales, y el tratamiento farmacológico (Tabla 1).

El tratamiento farmacológico se sustenta en el uso de anti-histamínicos (anti-H1) de $2^{\text {a }}$ generación, no obstante alrededor de $70 \%$ de los pacientes no logra mejoría completa ${ }^{23}$. Por este motivo se han estudiado y aprobado otro tipo de tratamientos para el manejo de UCE, como los fármacos inmunosupresores sistémicos y drogas biológicas.

\section{Anti-histamínicos}

La UCE es una enfermedad mediada por mastocitos, por lo que el tratamiento farmacológico se orienta a disminuir la liberación y los efectos de la histamina, así como de otros mediadores mastocitarios $^{1}$. Los anti-H1, han demostrado ser eficaces en disminuir el prurito, el número, tamaño, eritema y duración de los habones ${ }^{24}$. Debido a su buen perfil de seguridad y escasos efectos adversos, son los anti-H1 de $2^{\text {a }}$ generación los recomendados para tratar esta patología ${ }^{25}$. Además, estos fármacos son los únicos con evidencia clase 1 y recomendación grado A para el tratamiento 

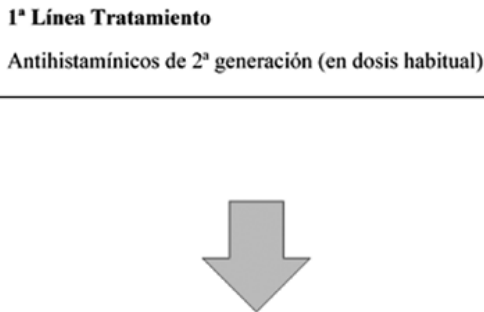

$2^{2}$ Línea Tratamiento

Aumentar dosis de antihistamínico de $2^{\mathrm{a}}$ generación hasta 4 veces

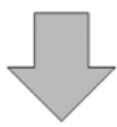

$3^{2}$ Linea Tratamiento

Agregar Omalizumab 300 mg se mensual

o

Ciclosporina $3-5 \mathrm{mg} / \mathrm{kg}$ día
Evitar gatillantes o factores

exacerbantes como AINES

Si persisten los sintomas después de 2-4 semanas (UAS7 $>7$ pts.)
Si persisten los sintomas

después de 2 semanas

(UAS7 > 7 pts)

Derivar al especialista
Figura 3. Algoritmo de tratamiento en urticaria crónica espontánea. de la UCE, lo cual está avalado por numerosos ensayos clínicos randomizados $(\mathrm{ECR})^{26}$. Hasta la fecha se han publicado distintos ECR con cetirizina, loratadina, desloratadina, fexofenadina, levocetirizina, bilastina y rupatadina en UCE, confirmando la seguridad y efectividad de estos fármacos en la disminución de síntomas y mejoría de la QoL en UCE ${ }^{26}$.

La recomendación es iniciar con un anti-H1 de $2^{\text {a }}$ generación a dosis habitual (1 dosis al día) siempre en forma diaria y no a demanda, ya que se ha demostrado una mayor efectividad en esta forma de administración ${ }^{27}$. En los casos refractarios se recomienda aumentar la dosis hasta cuatro veces al día (cuadriplicar la dosis diaria), evidenciándose su efectividad y seguridad en tratamientos a corto y largo plazo (meses hasta años) ${ }^{28}$.

No se recomienda mezclar distintos tipos de anti-H1 de 2a generación, ya que se ha observado que al cuadruplicar la dosis del mismo anti-H1 se potencia su efectividad ${ }^{28}$.
Los anti-H1 de $1^{\text {a }}$ generación (p. ej. clorfenamina, hidroxicina, doxepina y ciproheptadina), no están recomendados para el manejo de UCE debido a sus efectos adversos a nivel del sistema nervioso central (disminución del estado de alerta, sedación, alteración del ciclo sueño vigilia) y efectos anticolinérgicos (xerostomía, retención urinaria), tanto como tratamiento único como tampoco combinados con anti-H1 de $2^{\text {a }}$ generación ${ }^{25}$. Además no existen ECR que comprueben su efectividad y seguridad en $\mathrm{UCE}^{1}$.

\section{Anti-H1 en situaciones especiales}

Embarazo: Debido a aspectos éticos los datos con los que se cuenta provienen de estudios observacionales, por lo que no existen anti-H1 en categoría de seguridad A en embarazo, ni de $1^{\text {a }}$ ni de $2^{a}$ generación. Los anti-H1 de $2^{\text {a }}$ generación recomendados en embarazo tienen categoría B; éstos son loratadina, cetirizina y levocetirizina ${ }^{1}$. Si bien la clorfenamina también tiene categoría 
B en embarazo, presenta el riesgo de que en altas dosis puede tener un efecto oxitocínico por lo que debe evitarse en el tercer trimestre del embarazo ${ }^{25}$.

Lactancia: Todos los anti-H1 se excretan a bajas concentraciones en la leche materna, por lo que en esta condición se recomienda el uso de antihi-H1 de $2^{a}$ generación. No se recomiendan los anti-H1 de $1^{\text {a }}$ generación, ya que pueden producir sedación en el lactante ${ }^{1}$.

\section{Anti-leucotrienos (anti-LTRs)}

Si bien la histamina es uno de los principales mediadores liberados por mastocitos y basófilos activados, los leucotrienos juegan también un rol importante. Por este motivo se han realizado diversos estudios para probar el uso de anti-LTRs (montelukast, zafirlukast y pranlukast) en UCE.

Los primeros estudios demostraron eficacia de anti-LTRs sobre placebo en UCE, pero sin superioridad sobre anti-H1 de $2^{\text {a }}$ generación. El tratamiento con montelukast agregado a anti-H1 ha evidenciado resultados variables y poco concluyentes, con algunos ensayos que no demuestran mayor beneficio y otros que no demuestran mejoría ${ }^{29}$.

En suma, el nivel de evidencia es baja para recomendar el uso de montelukast en UCE, además este fármaco no tiene licencia para el tratamiento de UCE.

\section{Corticoesteroides}

En general no se recomienda el uso de corticoesteroides en UCE, sobre todo como terapia de largo plazo, debido a la gran cantidad de efectos adversos de estos fármacos ${ }^{1,23}$. Sin embargo, en situaciones puntuales, como una exacerbación del cuadro clínico se puede utilizar un pulso corto de prednisona en dosis de 20 a $50 \mathrm{mg}$ al día, por no más de 10 días ${ }^{23}$.

\section{Ciclosporina A}

Este inmunosupresor se encuentra aprobado para el tratamiento de la UCE y puede ser utilizado en aquellos pacientes que no han respondido a anti-H1 en dosis altas y/u omalizumab.

Numerosos ECR han demostrado que dosis bajas de ciclosporina (CsA) (1 a $3 \mathrm{mg} / \mathrm{kg} /$ día) logran una remisión completa de los síntomas y signos en $\mathrm{UCE}^{30}$. En un estudio, su uso por $12 \mathrm{se}-$ manas resultó en una mejoría completa de $68 \%$ de pacientes con $\mathrm{UC}^{31}$. Adicionalmente, se demostró utilidad en $2 / 3$ de los pacientes con UCE autoinmune refractarios a anti-H1, a dosis de $4 \mathrm{mg} / \mathrm{kg} / \mathrm{d}$ por 4-8 semanas, sin efectos adversos serios ${ }^{32}$.

Debido a los potenciales efectos adversos asociados a CsA, el uso de este medicamento requiere una monitorización frecuente. Dosis mayores a $5 \mathrm{mg} / \mathrm{k} /$ día, como las utilizadas en pacientes con trasplante, se han relacionado con aumento en el desarrollo de neoplasias, infecciones, alteraciones metabólicas, hipertensión arterial y nefrotoxici$\operatorname{dad}^{30}$.

\section{Hidroxicloroquina}

Existen pocos estudios en relación a la eficacia de este inmunomodulador como tratamiento de la UCE. Un ECR demostró una mejoría significativa en la QoL en pacientes con UC tratados con 200 $\mathrm{mg} /$ día de hidroxicloroquina durante 12 semanas, no obstante, los puntajes de actividad de la urticaria no cambiaron significativamente ${ }^{33}$.

Los eventos adversos más comunes son las alteraciones gastrointestinales. En cuanto al riesgo de retinopatía es bajo, llegando al $1 \%$ en los primeros 5 años de uso ${ }^{33}$.

A pesar de ser un fármaco con buen perfil de seguridad y bien tolerado, la calidad de la evidencia para su uso en UCE es muy baja, por lo que la fuerza de su recomendación es débil ${ }^{23}$.

\section{Metotrexato}

Los reportes del uso de metotrexato en UC son más bien anecdóticos y esporádicos, pese a ser un fármaco ampliamente utilizado en otras patologías cutáneas ${ }^{34}$.

Dada la falta de evidencia científica actual, no existe una recomendación respecto a su uso en UCE.

\section{Omalizumab}

Anticuerpo monoclonal humanizado recombinante anti-IgE que se une al fragmento Fc de la IgE libre, inhibiendo así su unión al receptor de alta afinidad de IgE (FceRI) expresado en la superficie de basófilos y mastocitos ${ }^{35}$.

Diversos estudios han demostrado la eficacia y seguridad de este fármaco en pacientes con UCE refractaria a terapia estándar con anti-H1. Un ECR multicéntrico con omalizumab a dosis de $150 \mathrm{mg}$ y 300 mg mensual, demostró mejoría significativa de la sintomatología de pacientes con UCE, la cual 
fue dosis dependiente ${ }^{33}$. El perfil de seguridad del fármaco fue comparable con placebo ${ }^{36}$.

Además, diferentes estudios han demostrado que omalizumab tiene una respuesta rápida y segura independiente de los niveles de IgE ni el peso del paciente ${ }^{37}$. Sin embargo, faltan estudios respecto a la duración ni forma de descenso de la terapia ${ }^{38}$.

En Chile, se encuentra aprobado en pacientes con UCE mayores de 12 años refractarios a terapia con anti-H1.

\section{Rituximab}

Anticuerpo monoclonal anti-CD20 que produce un efecto de inhibición sobre la producción de auto-anticuerpos. Existen algunos reportes sobre la utilidad terapéutica del Rituximab en UCE, pero no se han llevado a cabo ECR, por lo que la evidencia es de bajo grado para apoyar su uso ${ }^{39}$.

\section{Adalimumab, Etanercept, Infliximab}

Los antagonistas del factor de necrosis tumoral-alpha (TNF- $\alpha$ ) han sido ampliamente utilizados en otros trastornos inmunológicos. Algunos reportes de casos han mostrado eficacia en un pequeño número de pacientes, sin embargo, se necesitan más estudios clínicos controlados para demostrar su real beneficio terapéutico ${ }^{40}$.

\section{Gamaglobulina endovenosa}

Pequeñas series de casos ha evidenciado que gammaglobulina en dosis de $0,4 \mathrm{~g} / \mathrm{kg} /$ día por 5 días EV puede ser eficaz en pacientes con UCE; sin embargo, los autores señalan que su perfil costo-beneficio y posibles efectos adversos no la hace una terapia favorable en estos pacientes ${ }^{41}$.

\section{Tratamiento de urticaria crónica espontánea en niños}

La UCE es poco frecuente en niños, con una prevalencia de $1,8 \% \%^{42}$. Respecto al tratamiento, los anti-H1 de 2a generación son la primera opción, recomendaándose también escalar hasta cuadriplicar la dosis estándar según edad y peso $^{25}$. Una revisión Cochrane demostró que varios anti-H1 son eficaces y seguros en la supresión completa de la UCE, sin embargo, dado la falta de ECR, la tasa de respuesta en esta población no está clara, aunque es probable que sea similar a la de los adultos ${ }^{43}$.
En el último consenso EAACI/GA²LEN/EDF/ WAO publicado para el manejo de la urticaria se recomienda el uso exclusivo de medicamentos con eficacia y seguridad comprobada en pediatría, los cuales incluyen, cetirizina, desloratadina, fexofenadina, levocetirizina rupatadina, bilastina y loratadina ${ }^{44}$.

De segunda línea, aparecen ciclosporina a dosis de $3 \mathrm{mg} / \mathrm{k} / \mathrm{d}^{48}$ y Omalizumab en mayores de 12 años ${ }^{45,46}$.

\section{Conclusiones}

Esta guía ha sido confeccionada para establecer un criterio común entre los profesionales involucrados en el manejo de los pacientes adultos con UCE y así facilitar la terapia de esta patología en nuestro medio. No pretendía ser una revisión exhaustiva o un esquema rígido de aplicación de los conocimientos científicos en el paciente individual, ya que cada paciente presenta características clínicas propias, que pueden llevar a tomar decisiones diferentes de las aquí enunciadas. Del mismo modo, la aplicación en su totalidad de las recomendaciones de la presente guía no garantiza el éxito en todos los pacientes.

Se procuró, para cada una de las recomendaciones, considerar la mejor evidencia disponible en la literatura. En aquellas situaciones en que no se contaba con esta información, se recurrió a lo reportado en los consensos de expertos. También se consideró la experiencia de cada uno de los miembros de este grupo de trabajo, ya que sin duda, algunas de las prácticas clínicas habituales han sido evidentemente útiles.

Se tuvo presente que la ausencia de evidencia respecto de algún procedimiento o actuar médico no necesariamente proscribe su utilización, ya que en algunos casos no es posible ir más lejos en la investigación o se está limitado por razones éticas.

Material suplementario en versión digital de la Revista Médica de Chile en: http:/www.scielo.cl

\section{Referencias}

1. Zuberbier T, Aberer W, Asero R, Bindslev-Jensen C, Brzoza Z, Canonica GW, et al. The EAACI/GA(2) LEN/ $\mathrm{EDF} / \mathrm{WAO}$ Guideline for the definition, classification, 
diagnosis, and management of urticaria: the 2013 revision and update. Allergy 2014; 69: 868-87.

2. Dreyfus DH. Differential Diagnosis of Chronic Urticaria and Angioedema Based on Molecular Biology, Pharmacology, and Proteomics. Immunol Allergy Clin North Am 2017; 37: 201-15.

3. Magerl M, Borzova E, Giménez-Arnau A, Grattan CE, Lawlor F, Mathelier-Fusade P, et al. The definition and diagnostic testing of physical and cholinergic urticarias--EAACI/GA2LEN/EDF/UNEV consensus panel recommendations. Allergy 2009; 64: 1715-21.

4. Zuberbier T, Balke M, Worm M, Edenharter G, Maurer M. Epidemiology of urticaria: a representative cross-sectional population survey. Clin Exp Dermatol 2010; 35 : 869-73.

5. Maurer M, Magerl M, Metz M, Zuberbier T. Revisions to the international guidelines on the diagnosis and therapy of chronic urticaria. J Dtsch Dermatol Ges 2013; 11: 971-7; quiz 978.

6. Tedeschi A, Kolkhir P, Asero R, Pogorelov D, Olisova O, Kochergin N, et al. Chronic urticaria and coagulation: pathophysiological and clinical aspects. Allergy 2014; 69: 683-91.

7. Sabroe RA GC, Francis DM, Barr RM, Kobza Black A, Greaves MW. The autologous serum skin test: a screening test for autoantibodies in chronic idiopathic urticaria. British Journal of Dermatology 1999; 446-52.

8. Mlynek A, Zalewska-Janowska A, Martus P, Staubach $\mathrm{P}$, Zuberbier T, Maurer M. How to assess disease activity in patients with chronic urticaria? Allergy 2008; 63: 777-80.

9. Weller K, Groffik A, Magerl M, Tohme N, Martus P, Krause $\mathrm{K}$, et al. Development, validation, and initial results of the Angioedema Activity Score. Allergy 2013; 68: 1185-92.

10. García-Diez I, Curto-Barredo L, Weller K, Pujol RM, Maurer M, Giménez-Arnau AM. Cross-Cultural Adaptation of the Urticaria Control Test From German to Castilian Spanish. Actas Dermosifiliogr 2015; 106: 74652.

11. Hamad A, Jithpratuck W, Krishnaswamy G. Urticarial vasculitis and associated disorders. Ann Allergy Asthma Immunol 2017; 118: 394-8.

12. Valent P, Akin C, Hartmann K, Nilsson G, Reiter A, Hermine $\mathrm{O}$, et al. Advances in the Classification and Treatment of Mastocytosis: Current Status and Outlook toward the Future. Cancer Res 2017; 77: 1261-70.

13. Taylor D, Pappo E, Aronson IK. Polymorphic eruption of pregnancy. Clin Dermatol 2016; 34: 383-91.

14. Lamoreux MR, Sternbach MR, Hsu WT. Erythema Multiforme. American Family Physician 2017; 74: 1883-8.
15. Brodell LA, Beck LA. Differential diagnosis of chronic urticaria. Ann Allergy Asthma Immunol 2008; 100: 1818; quiz 188-190, 215.

16. Engin B, Uguz F, Yilmaz E, Ozdemir M, Mevlitoglu I. The levels of depression, anxiety and quality of life in patients with chronic idiopathic urticaria. J Eur Acad Dermatol Venereol 2008; 22: 36-40.

17. Both H, Essink-Bot ML, Busschbach J, Nijsten T. Critical review of generic and dermatology-specific health-related quality of life instruments. J Invest Dermatol 2007; 127: 2726-39.

18. Baiardini I, Pasquali M, Braido F, Fumagalli F, Guerra L, Compalati E, et al. A new tool to evaluate the impact of chronic urticaria on quality of life: chronic urticaria quality of life questionnaire (CU-QoL). Allergy 2005; 60: 1073-8.

19. Valero A, Herdman M, Bartra J, Ferrer M, Jauregui I, Davila I, et al. Adaptation and validation of the Spanish version of the Chronic Urticaria Quality of Life Questionnaire (CU-Q2oL). J Investig Allergol Clin Immunol 2008; 18: 426-32.

20. Weller K, Groffik A, Church MK, Hawro T, Krause $\mathrm{K}$, Metz $\mathrm{M}$, et al. Development and validation of the Urticaria Control Test: a patient-reported outcome instrument for assessing urticaria control. J Allergy Clin Immunol 2014; 133: 1365-72, 1372.e1361-1366.

21. Silvares MR, Fortes MR, Miot HA. Quality of life in chronic urticaria: a survey at a public university outpatient clinic, Botucatu (Brazil). Rev Assoc Med Bras (1992) 2011; 57: 577-82.

22. Staubach P, Dechene M, Metz M, Magerl M, Siebenhaar F, Weller K, et al. High prevalence of mental disorders and emotional distress in patients with chronic spontaneous urticaria. Acta Derm Venereol 2011; 91: 557-61.

23. Vestergaard C, Toubi E, Maurer M, Triggiani M, Ballmer-Weber B, Marsland A, et al. Treatment of chronic spontaneous urticaria with an inadequate response to H1-antihistamines: an expert opinion. Eur J Dermatol 2017; 27: 10-9.

24. Simons FE, Silver NA, Gu X, Simons KJ. Clinical pharmacology of H1-antihistamines in the skin. J Allergy Clin Immunol 2002; 110: 777-83.

25. Church MK, Maurer M, Simons FE, Bindslev-Jensen C, van Cauwenberge P, Bousquet J, et al. Risk of first-generation $\mathrm{H}(1)$-antihistamines: a $\mathrm{GA}(2) \mathrm{LEN}$ position paper. Allergy 2010; 65: 459-66.

26. Kavosh ER, Khan DA. Second-generation H1-antihistamines in chronic urticaria: an evidence-based review. Am J Clin Dermatol 2011; 12: 361-76.

27. Weller K, Ardelean E, Scholz E, Martus P, Zuberbier T, Maurer M. Can on-demand non-sedating antihis- 
tamines improve urticaria symptoms? A double-blind, randomized, single-dose study. Acta Derm Venereol 2013; 93: 168-74.

28. Guillen-Aguinaga S, Jauregui Presa I, Aguinaga-Ontoso E, Guillen-Grima F, Ferrer M. Updosing nonsedating antihistamines in patients with chronic spontaneous urticaria: a systematic review and meta-analysis. $\mathrm{Br} \mathrm{J}$ Dermatol 2016; 175: 1153-65.

29. Di Lorenzo G, Pacor ML, Mansueto P, Esposito Pellitteri M, Lo Bianco C, Ditta V, et al. Randomized placebo-controlled trial comparing desloratadine and montelukast in monotherapy and desloratadine plus montelukast in combined therapy for chronic idiopathic urticaria. J Allergy Clin Immunol 2004; 114: 619-25.

30. Kulthanan K, Chaweekulrat P, Komoltri C, Hunnangkul S, Tuchinda P, Chularojanamontri L, et al. Cyclosporine for Chronic Spontaneous Urticaria: A Meta-Analysis and Systematic Review. J Allergy Clin Immunol Pract 2017.

31. Toubi E, Blant A, Kessel A, Golan TD. Low-dose cyclosporin $A$ in the treatment of severe chronic idiopathic urticaria. Allergy 1997; 52: 312-6.

32. Grattan CE, O’Donnell BF, Francis DM, Niimi N, Barlow RJ, Seed PT, et al. Randomized double-blind study of cyclosporin in chronic 'idiopathic' urticaria. Br J Dermatol 2000; 143: 365-72.

33. Reeves GE, Boyle MJ, Bonfield J, Dobson P, Loewenthal M. Impact of hydroxychloroquine therapy on chronic urticaria: chronic autoimmune urticaria study and evaluation. Intern Med J 2004; 34: 182-6.

34. Sharma VK, Singh S, Ramam M, Kumawat M, Kumar R. A randomized placebo-controlled double-blind pilot study of methotrexate in the treatment of $\mathrm{H} 1$ antihistamine-resistant chronic spontaneous urticaria. Indian J Dermatol Venereol Leprol 2014; 80: 122-8.

35. Cooke A, Bulkhi A, Casale TB. Role of biologics in intractable urticaria. Biologics 2015; 9: 25-33.

36. Maurer M, Rosen K, Hsieh HJ, Saini S, Grattan C, Gimenez-Arnau A, et al. Omalizumab for the treatment of chronic idiopathic or spontaneous urticaria. N Engl J Med 2013; 368: 924-35.

37. Metz M, Ohanyan T, Church MK, Maurer M. Omali- zumab is an effective and rapidly acting therapy in difficult-to-treat chronic urticaria: a retrospective clinical analysis. J Dermatol Sci 2014; 73: 57-62.

38. Kaplan A, Ledford D, Ashby M, Canvin J, Zazzali JL, Conner E, et al. Omalizumab in patients with symptomatic chronic idiopathic/spontaneous urticaria despite standard combination therapy. J Allergy Clin Immunol 2013; 132: 101-9.

39. Boyman O, Kaegi C, Akdis M, Bavbek S, Bossios A, Chatzipetrou A, et al. EAACI IG Biologicals task force paper on the use of biologic agents in allergic disorders. Allergy 2015; 70: 727-54.

40. Wilson LH, Eliason MJ, Leiferman KM, Hull CM, Powell DL. Treatment of refractory chronic urticaria with tumor necrosis factor-alfa inhibitors. J Am Acad Dermatol. United States; 2011: p. 1221-2.

41. Watkins C, Peiris E, Saleh H, Krishnaswamy G. Intravenous immunoglobulin as a potential therapy for refractory urticaria - a review. Inflamm Allergy Drug Targets 2012; 11: 375-81.

42. Lee SJ, Ha EK, Jee HM, Lee KS, Lee SW, Kim MA, et al. Prevalence and Risk Factors of Urticaria With a Focus on Chronic Urticaria in Children. Allergy Asthma Immunol Res 2017; 9: 212-9.

43. Sharma M, Bennett C, Carter B, Cohen SN. H1-antihistamines for chronic spontaneous urticaria: an abridged Cochrane Systematic Review. J Am Acad Dermatol 2015; 73: 710-6.e714.

44. Zuberbier T, Aberer W, Asero R, Abdul Latiff AH, Baker D, Ballmer-Weber B, et al. The EAACI/GA(2)LEN/ $\mathrm{EDF} / \mathrm{WAO}$ guideline for the definition, classification, diagnosis and management of urticaria. Allergy 2018; 73: 1393-414.

45. Doshi DR, Weinberger MM. Experience with cyclosporine in children with chronic idiopathic urticaria. Pediatr Dermatol 2009; 26: 409-13.

46. Saini S, Rosen KE, Hsieh HJ, Wong DA, Conner E, Kaplan A, et al. A randomized, placebo-controlled, dose-ranging study of single-dose omalizumab in patients with $\mathrm{H} 1$-antihistamine-refractory chronic idiopathic urticaria. J Allergy Clin Immunol 2011; 128: 567-73. e561. 